\title{
Effectiveness of Cognitive-behavioral Therapy on Psychological Well-being in Infertile Women: A Randomized Controlled Trial
}

\author{
Zahra Marashi ${ }^{1}$, Nasser Behroozi ${ }^{2}$, Mohammad H Haghighi-Zadeh ${ }^{3}$, Roshan Nikbakht ${ }^{4}$, Zahra Abbaspoor ${ }^{5}$
}

\section{Abstract}

Background: Psychological well-being is a psychological variable that affects infertile women. This study aimed to explore the effect of cognitivebehavioral therapy (CBT) on the psychological well-being of infertile women.

Materials and methods: Sixty-eight women with infertility randomly allocated into two groups: CBT $(n=34)$ and control $(n=34)$ groups. The women in the CBT program were divided into two subgroups of 12 and one group of 10, with each receiving eight therapy sessions (each session lasting 90 minutes). Psychological well-being was measured using a demographic questionnaire and the short-form Ryff Psychological well-being scale. The independent and paired $t$-tests, Chi-square, repeated measures, and ANCOVA tests were used to analyze the data.

Results: Two women from the CBT and three from the control groups withdrew from the study. In the CBT group, the general psychological wellbeing score and self-acceptance, positive relationship, autonomy, purpose in life, personal growth, and environmental mastery dimensions'scores were improved after the intervention. The scores also were significantly higher in the case group than those in the control group $(p<0.001)$. Conclusion: Cognitive-behavioral therapy can improve all aspects of psychological well-being, including self-acceptance, positive relationships, autonomy, purpose in life, personal growth, and environmental mastery in infertile women.

Trial registration number: IRCT20190311043019N1.

Keywords: Cognitive-behavioral therapy, Infertile women, Psychological well-being.

International Journal of Infertility and Fetal Medicine (2021): 10.5005/jp-journals-10016-1220
\end{abstract}

\section{INTRODUCTION}

Infertility is defined as a failure in pregnancy after one year of intercourse without the use of any contraceptive method. ${ }^{1}$ Many people consider infertility the saddest and the most stressful experience of life and describe repeated periods of treatment as repeated periods of crisis. ${ }^{2}$ More than 50 million couples worldwide are suffering from infertility. ${ }^{3}$ The experience of infertility can lead to psychiatric disorders such as depression, anxiety, helplessness, interpersonal relationship problems, low self-acceptance, low self-confidence, isolation, and identity issues. ${ }^{4}$ In Iran, psychiatric disorders and personality instability in infertile women are approximately twice that of fertile women and these women have lower psychological well-being. ${ }^{5}$ Psychological well-being is a psychological variable that affects infertile women. ${ }^{6}$ There are different types of well-being, including subjective, emotional, social, spiritual, and psychological well-being. ${ }^{7}$ Mental well-being is defined by happiness, pleasant success, and pain avoidance, ${ }^{8}$ but the psychological well-being requires an understanding of the challenges of life, and the growth and development in face of them, and places great emphasis on human development. ${ }^{9}$

Psychological well-being is considered a multi-dimensional structure consisting of cognitive and emotional components. ${ }^{10}$ The well-being cognitive dimension is described as an individual's cognitive assessment about her life satisfaction rate and the emotional dimension is defined as feeling high positive emotions and low negative emotions. ${ }^{11}$ Individuals with higher psychological wellbeing try to flourish their abilities consistently, usually experience positive emotions, and have positive evaluations about the

\begin{abstract}
1,5 Department of Midwifery, Reproductive Health Promotion Research Center, Ahvaz Jundishapur University of Medical Sciences, Ahvaz, Iran ${ }^{2}$ Department of Psychology, Shahid Chamran University of Ahvaz, Ahvaz, Iran

${ }^{3}$ Department of Biostatistics, School of Public Health, Ahvaz Jundishapur University of Medical Sciences, Ahvaz, Iran

${ }^{4}$ Department of Obstetrics and Gynecology, Fertility, Infertility and Perinatology Research Center, Ahvaz Jundishapur University of Medical Sciences, Ahvaz, Iran

Corresponding Author: Zahra Abbaspoor, Department of Midwifery, Reproductive Health Promotion Research Center, Ahvaz Jundishapur University of Medical Sciences, Ahvaz, Iran, Phone: +9833738331, e-mail: Abbaspoor_z762@yahoo.com
\end{abstract}

How to cite this article: Marashi Z, Behroozi N, Haghighi-Zadeh $\mathrm{MH}$, et al. Effectiveness of Cognitive-behavioral Therapy on Psychological Well-being in Infertile Women: A Randomized Controlled Tria. Int J Infertil Fetal Med 2021;12(2):25-30.

Source of support: This article is extracted from Master's thesis of Zahra Marashi, and the research project with number: RHPRC-9717. This research was supported by the vice-chancellor for research of Ahvaz Jundishapur University of Medical Sciences.

Conflict of interest: None

surrounding events. High psychological well-being is an important factor in growth, health, and the prevention of psychological disorders. But low psychological well-being leads to a greater perception of negative emotions, including depression, anxiety, violence, low self-acceptance, social problems, and interpersonal

(c) Jaypee Brothers Medical Publishers. 2021 Open Access This article is distributed under the terms of the Creative Commons Attribution 4.0 International License (https://creativecommons.org/licenses/by-nc/4.0/), which permits unrestricted use, distribution, and non-commercial reproduction in any medium, provided you give appropriate credit to the original author(s) and the source, provide a link to the Creative Commons license, and indicate if changes were made. The Creative Commons Public Domain Dedication waiver (http://creativecommons.org/publicdomain/zero/1.0/) applies to the data made available in this article, unless otherwise stated. 
problems. ${ }^{10}$ Infertility is associated with low psychological wellbeing that can lead to feelings of inferiority, suppressed anger, guilt, hopelessness, and marital and sexual problems. These couples have deep emotional tensions and psychological and social fears and suffer from anxiety and depression and also have less selfconfidence. ${ }^{5}$ Effective psychological interventions for maintaining and promoting psychological well-being include many items; for instance: acceptance and commitment therapy, couple therapy, and mindfulness therapy, which reduce the stress of infertility and increase positive thoughts in infertile women.

Another psychological intervention is cognitive-behavioral therapy (CBT) which is considered a combination of therapeutic cognitive and behavioral techniques. ${ }^{12}$ This therapeutic method focuses on the relationship between thinking, emotion, and behavior and emphasizes the central role of thinking and its related processes. Cognitive therapies encourage the patients to test the relationship between their negative automatic thoughts and their feelings and consider behaviors that are the results of negative thoughts as a criterion for evaluating those thoughts. ${ }^{13}$ Studies have emphasized the various effects of CBT on psychological functions. Ahmadi et al. showed that CBT leads to the improvement of mental image in infertile women. ${ }^{14}$ Also, Mosalanejad et al. showed that CBT improves mental health and hardiness in infertile women. ${ }^{15}$ Besides Talaei et al showed in their study that CBT reduces depression in infertile women. ${ }^{16}$ Other research has shown that CBT improves psychological well-being in women with other problems, including breast cancer by improving their sexual identity and their self-acceptance, ${ }^{17}$ and in cardiac diseases by improving their personal growth and their purpose in life. ${ }^{18}$ Further studies concerning the frequency of psychiatric disorders in infertile women and the importance of mental health and its possible impact on the results of infertility treatment are essential. ${ }^{19}$ The present study intended to investigate the effect of CBT on the psychological well-being of infertile women.

\section{Materials and Methods}

This randomized controlled trial study was carried out among infertile women attending the antenatal infertility clinic of Imam Khomeini Hospital in Ahvaz, Iran, between April and September 2019. The participants were recruited as per inclusion and exclusion criteria.

Inclusion criteria were including the statistical population of this study was infertile women referred to the infertility clinic of Imam Khomeini Hospital in Ahvaz City in 2019. Inclusion criteria were included:age 20-45 years, primary infertility, ability to read and write, infertility-related stress based on a score lower than the average of the psychological well-being questionnaire. Exclusion criteria were including pregnancy, severe mental illness that requires medication, such as major depression and dementia or other severe mental disorders, more than twice absenteeism in counseling sessions, having an adopted child, and having a history of stressful life events in the past 6 months that lead to hospitalization in a psychiatric ward based on the information in the patient's medical records.

The protocol of this study was approved by the ethics committee of the Ahvaz Jundishapur University of Medical Sciences, (IR.AJUMS.REC.1397.931). The objectives of the study were explained to the participants and they were assured about confidentiality; also, informed consent (oral and written) were obtained from all the participants. This study was also registered in the Iranian registry of clinical trials (registration code: IRCT20190311043019N1).

\section{Sampling Method}

The sample size was calculated using the formula below ${ }^{17}$ :

$$
\begin{gathered}
n=\frac{\left(z_{1}-\frac{\alpha}{2}+z_{1-\beta}\right)^{2}\left(s_{1}^{2}+s_{2}^{2}\right)}{\left(\bar{x}_{1}-\bar{x}_{2}\right)^{2}} \\
\frac{(1.96+0.84)^{2}\left(4.61^{2}+2.96\right)^{2}}{(66.8-64.06)^{2}}=31
\end{gathered}
$$

The study's power was set at $90 \%$ and $\alpha=0.01, \mathrm{~S} 1=4.61$, $\mathrm{S} 2=2.96, \bar{x}_{1}=66.8, \bar{x}_{2}=64.06,{ }_{1-\frac{\alpha}{2}}=1.96, z_{1-\beta}=1.28$ and $z_{1-\beta}=0.84$.

The sample size was estimated to be 34 in each group based on a $10 \%$ attrition risk.

\section{Measures}

The data were collected using a demographic questionnaire and the Ryff Psychological Well-being Scale's 18-item short-form. The demographic questionnaire asked about age, spouse's age, work, schooling, length of the marriage, and infertility background.

Ryff developed the Ryff Psychological Well-being Questionnaire in 1989 and updated it in 2002. The main form of the questionnaire included 120 questions, but the 18 questions type was used in this study. The Likert score range is between 1 (I totally disagree) and 6 (I totally agree). Ten questions are directly scored and eight questions are scored in reverse. The lowest score is 18 and the highest is 108 . This questionnaire contains six components and the total score. A higher score equals higher psychological well-being. The components of the Ryff questionnaire are including self-acceptance, positive relationship, autonomy, purpose in life, personal growth, and environmental mastery. The validity and reliability of the Ryff Psychological Well-being scale were confirmed by Keyes et al. with Cronbach's $\alpha$ measured at $0.89,{ }^{6}$ and in Iran, Khanjani et al. have confirmed it, with a Cronbach's $\alpha$ of $0.71 .^{20}$

\section{Randomization}

Women who were qualified and gave their consent were randomly allocated to one of two groups: CBT or control. We used block randomization with a block size of 4 and a 1:1 allocation ratio. Every woman was given a code for allocation concealment, which was stored in an invisible sealed envelope by the clinic secretary until the start of the counseling.

\section{Intervention}

The CBT group received eight weeks of counseling focused on $\mathrm{CBT}^{21}$ (one session every week), while the control group received no intervention. Two groups of 12 and one group of 10 participated in the counseling sessions. Each session lasted 90 minutes. In each session, one researcher (ZM) first reviewed the concepts and homework of the previous session and then presented new content and tasks. A summary of the content of the treatment sessions is given in Table 1. At the end of the intervention, the control group obtained one CBT compact disk (CD). In this study, blinding the researcher and participants was not possible. 


\section{Statistical Analyses}

SPSS Inc. USA, Chicago, Illinois, version 23 was used to enter all of the data. Missing values were imputed by multiple imputation procedures. The Kolmogorov-Smirnov test was used to assess the normality of the data distribution. The independent $t$-test or Mann-Whitney test was used to compare continuous data between the two groups, and the Chi-square test was used to compare categorical data. To compare continuous data within the groups, the paired $t$-test and repeated measures tests were used. To neutralize confounding effects, an analysis of covariance was used. In this analysis, the test power was $90 \%$, and the level of significance was set at $p<0.05$.

\section{Outcome}

The outcome was to evaluate the effect of CBT on the psychological well-being of infertile women.

\section{Results}

In each group, 34 women were recruited; however, two women from the case group withdrew from the study, one due to pregnancy and the other due to absenteeism. In addition, three women in the control group dropped out, two due to pregnancy and the other for personal reasons. Flowchart 1 shows the recruitment and retention of women.

In terms of age, spouse's age, marriage length, infertility duration, unsuccessful IUI times, unsuccessful ART times, education, occupation, and infertility factor, there was no significant difference between the two groups $(p>0.05)$. In terms of duration of treatment, there was a significant difference between the two groups ( $p=0.01$ ) (Table 2). To neutralize confounding effects, an analysis of covariance was used.

Table 3 shows different domains of psychological well-being before and after the intervention in two groups of CBT and control. The scores of self-acceptance, positive relationship, autonomy, purpose in life, personal growth, and environmental mastery were significantly improved in the CBT group after the intervention, compared to the control group ( $p<001)$. Overall psychological well-being significantly improved in the CBT group as compared to the control group $(p<001)$.

\section{Discussion}

This research aimed to see the effect of CBT on the psychological well-being of infertile women. The results showed that there was a significant difference between the two groups in terms of duration of treatment. Prolonged infertility treatment increases psychiatric problems. ${ }^{16}$ The findings revealed that CBT improved self-acceptance in infertile women. In other words, CBT helped reinforce the self-acceptance dimension by training the cognitive restructuring and self-esteem skills. ${ }^{13}$ This result was in line with the findings of Bavadi et al. which states that CBT helps women who suffer from breast cancer to love themselves as they are. ${ }^{17}$

Table 1: Session outlines for the cognitive-behavioral therapy (CBT) group treatment protocol

\begin{tabular}{ll}
\hline First session & $\begin{array}{l}\text { Introduce participants, follow rules, discussing } \\
\text { the connection between psychological well- } \\
\text { being and infertility }\end{array}$ \\
Second session & $\begin{array}{l}\text { How to use the CBT model to reduce infertil- } \\
\text { ity stress, identification of automated negative } \\
\text { thoughts }\end{array}$ \\
Third session & $\begin{array}{l}\text { Evaluation of automated negative thoughts } \\
\text { Challenging the automatic negative thoughts } \\
\text { Fourth session }\end{array}$ \\
Fifth session & $\begin{array}{l}\text { Determining the relationship between thought } \\
\text { and feeling and behavior in situations, nuclear } \\
\text { beliefs }\end{array}$ \\
Sixth session & $\begin{array}{l}\text { Nuclear beliefs about infertility } \\
\text { Changing coping strategies }\end{array}$ \\
Seventh session & $\begin{array}{l}\text { Instructions on meditation, coping with } \\
\text { ruminations-obsessions-worrying, expressive } \\
\text { writing, and assertive communication }\end{array}$ \\
\hline
\end{tabular}

Flowchart 1: Flowchart of recruitment and retention of women in the study

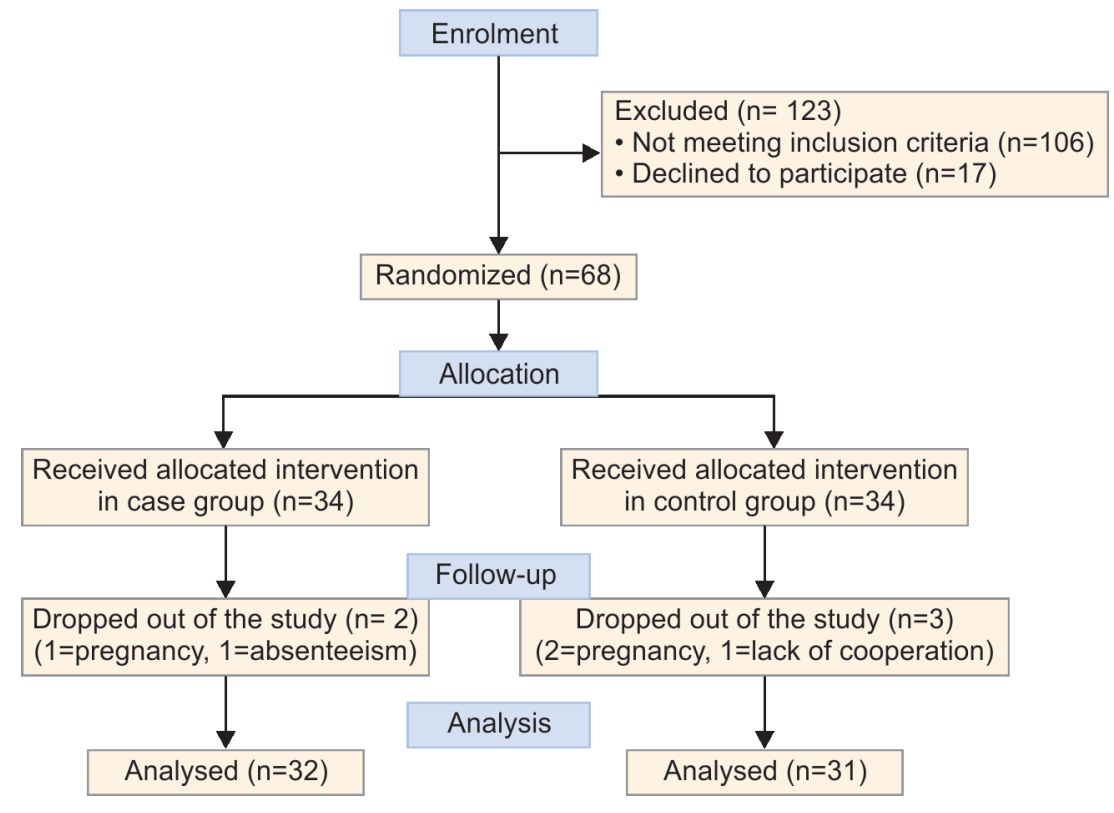


Table 2: The sociodemographic characteristics of infertile women in the case and control groups

\begin{tabular}{|c|c|c|c|c|}
\hline \multicolumn{2}{|l|}{ Characteristics } & $p$ value & Control group $(n=34)$ & Case group $(n=34)$ \\
\hline \multicolumn{2}{|l|}{ Age } & $31.3(5.5)$ & $31(4.7)$ & 0.80 \\
\hline \multicolumn{2}{|l|}{ Spouse age } & $34.1(5.6)$ & $33.6(4.6)$ & 0.80 \\
\hline \multicolumn{2}{|l|}{ Marriage duration, years } & $6.3(3.1)$ & $5.9(3.2)$ & 0.60 \\
\hline \multicolumn{2}{|l|}{ Infertility duration, years } & $5.1(2.8)$ & $4.2(2.9)$ & 0.21 \\
\hline \multicolumn{2}{|c|}{ Duration of treatment, years } & $3.5(2.1)$ & $2.3(1.5)$ & 0.01 \\
\hline \multicolumn{2}{|c|}{ Unsuccessful IUI times } & $0.58(0.9)$ & $0.56(1.2)$ & 0.90 \\
\hline \multicolumn{2}{|l|}{ Unsuccessful ART times } & $0.17(0.3)$ & $0.19(0.5)$ & 0.79 \\
\hline \multirow[t]{4}{*}{ Education attainment } & High school & $14(41.18)$ & $11(32.35)$ & 0.23 \\
\hline & Diploma & $16(47.05)$ & $14(41.18)$ & \\
\hline & Bachelor & $3(8.82)$ & $9(26.47)$ & \\
\hline & Postgraduate & $1(2.95)$ & $0(0.0)$ & \\
\hline \multirow[t]{2}{*}{ Employment } & Employee & $30(88.23)$ & 29 (85.29) & 0.42 \\
\hline & Housewife & $4(11.77)$ & $5(14.71)$ & \\
\hline \multirow[t]{4}{*}{ Infertility factor } & Wife & $6(17.65)$ & $7(20.30)$ & 0.73 \\
\hline & Husband & $18(52.94)$ & $14(41.19)$ & \\
\hline & Both & $7(20.59)$ & $10(29.42)$ & \\
\hline & None & $3(8.82)$ & $3(8.82)$ & \\
\hline
\end{tabular}

Values are presented as mean (SD) and $n(\%)$

Table 3: Psychological well-being within and between groups before and after the intervention (immediately and 1 month later)

\begin{tabular}{|c|c|c|c|c|c|}
\hline Psychological well-being & Time & Case group $(n=32)$ & Control group $(n=31)$ & $p$ value ${ }^{1}$ & $p$ value ${ }^{2}$ \\
\hline \multirow[t]{5}{*}{ Self-acceptance } & Pretest & $9.9(0.9)$ & $9.8(1.7)$ & 0.168 & $<0.001$ \\
\hline & Posttest & $12.3(1.0)$ & $10.0(1.6)$ & $<0.001$ & \\
\hline & Follow-up & $12.2(0.9)$ & $9.6(1.6)$ & $<0.001$ & \\
\hline & $p$ value $^{2}$ & $<0.001$ & 0.052 & & \\
\hline & $p$ value $^{3}$ & $<0.001$ & 0.336 & & \\
\hline \multirow[t]{10}{*}{ Positive relationship } & Pretest & $10.1(1.4)$ & $10.1(1.6)$ & 0.816 & $<0.001$ \\
\hline & Posttest & $12.2(1.2)$ & $9.8(1.5)$ & $<0.001$ & $<0.001$ \\
\hline & Follow-up & $12.2(1.1)$ & $9.9(1.4)$ & $<0.001$ & \\
\hline & $p$ value $^{2}$ & $<0.001$ & 0.368 & 0.274 & \\
\hline & $p$ value $^{3}$ & $<0.001$ & 0.083 & $<0.001$ & \\
\hline & Pretest & $10.0(1.2)$ & $9.5(1.6)$ & $<0.001$ & \\
\hline & Posttest & $12.1(1.3)$ & $9.5(1.8)$ & & \\
\hline & Follow-up & $12.1(1.4)$ & $9.5(1.3)$ & & \\
\hline & $p$ value $^{2}$ & $<0.001$ & 0.764 & & \\
\hline & $p$ value $^{3}$ & $<0.001$ & 0.624 & & \\
\hline \multirow[t]{5}{*}{ Purpose in life } & Pretest & $10.5(1.2)$ & $10.1(1.4)$ & 0.288 & $<0.001$ \\
\hline & Posttest & $12.5(1.1)$ & $10.3(1.6)$ & $<0.001$ & \\
\hline & Follow-up & $12.4(1.2)$ & $10.1(1.2)$ & $<0.001$ & \\
\hline & $p$ value $^{2}$ & $<0.001$ & 0.880 & & \\
\hline & $p$ value $^{3}$ & $<0.001$ & 0.246 & & \\
\hline \multirow[t]{5}{*}{ Environmental mastery } & Pretest & $9.3(1.4)$ & $9.9(1.4)$ & 0.160 & $<0.001$ \\
\hline & Posttest & $11.6(1.3)$ & $9.7(1.5)$ & $<0.001$ & \\
\hline & Follow-up & $11.6(1.2)$ & $9.7(1.3)$ & $<0.001$ & \\
\hline & $p$ value $^{2}$ & $<0.001$ & 0.663 & & \\
\hline & $p$ value $^{3}$ & $<0.001$ & 0.478 & & \\
\hline \multirow[t]{5}{*}{ Psychological well-being } & Pretest & $60.1(1.0)$ & $59.8(1.0)$ & 0.168 & $<0.001$ \\
\hline & Posttest & $73.2(2.9)$ & $59.6(1.6)$ & $<0.001$ & \\
\hline & Follow-up & $73.2(3.1)$ & $58.9(1.0)$ & $<0.001$ & \\
\hline & $p$ value $^{2}$ & $<0.001$ & 0.106 & & \\
\hline & $p$ value $^{3}$ & $<0.001$ & 0.350 & & \\
\hline
\end{tabular}

Values are presented as mean (SD)

${ }^{1}$ Independent $t$-test

${ }^{2}$ Repeated measures

${ }^{3}$ Paired $t$-test (pretest/posttest) 
Cognitive-behavioral therapy was also effective in improving positive relationships with others in infertile women significantly. Cognitive-behavioral therapy helped women to effectively deal with others through communication training and self-control skills. ${ }^{22}$ Roothman et al. reported that CBT could enhance the social aspects of psychological well-being such as positive relationships with others. ${ }^{23}$ In the present study, we found that CBT positively affected the autonomy dimension of infertile women. Cognitivebehavioral therapy led to a reinforcement of autonomy through training for cognitive restructuring. ${ }^{6}$ It is in line with the findings of Faramarzi et al.'s study which states that CBT is effective in decreasing fertility stress in dimensions of the social concerns, restructuring, and eliminating negative automatic thoughts, and improving autonomy. ${ }^{24}$ Also, the present study demonstrated that CBT is effective in enhancing infertile women's sense of meaning, which is in line with the findings of Faramarzi et al.'s study. ${ }^{24}$ Cognitive-behavioral therapy enhances purposefulness through decision-making skills and understanding the consequences. This observation is consistent with the findings of Abdolahi et al. which showed that CBT expands conscious imagination and increases purposefulness in infertile women suffering from anxiety and depression. ${ }^{25}$ Additionally, CBT improved the personal growth of infertile women. This finding is consistent with the results of Keyes et al.'s study which found that CBT improved self-efficacy and helped personal growth. ${ }^{6}$ Also, Abdolahi et al. showed that CBT could greatly increase infertile women's personal growth. ${ }^{25}$ The present study also showed that CBT contributed to improving the environmental mastery in infertile women. It also helped individuals create the desired environment via problem-solving skills and interaction. ${ }^{24}$ This finding is consistent with the results of Hamzeh Pour's study which found that CBT enhances interaction and environmental mastery and reduces the level of anxiety, depression, and other psychological problems by focusing on attitude, identifying and correcting cognitive distortions and thoughts for infertile women suffering from anxiety. ${ }^{26}$

Overall, the results of this study showed that CBT is effective in improving psychological well-being in infertile women. To explain, the CBT emphasizes on awareness of cognitive distortions and its destructive effects. The therapist helps the patient to identify and modify their irrational beliefs. ${ }^{18}$ The results of the present study are consistent with Espie et al.'s study that found CBT leads to improving psychological well-being and quality of life in patients who suffered from insomnia. ${ }^{27}$

\section{The Study's Strengths and Limitation}

This is the first research to look at the impact of CBT on psychological well-being in infertile women with a 1-month follow-up. However, there are some limitations to this study. First, recall bias can influence responses to psychological well-being questionnaire questions. Second, this study was not blinded, and neither the researchers nor the women knew which group they were in until the intervention.

\section{Conclusion}

This study found that CBT can improve all aspects of psychological well-being, including self-acceptance, positive relationships, autonomy, purpose in life, personal growth, and environmental mastery dimensions in women with infertility. More research with a longer follow-up period is suggested.

\section{References}

1. Gorayeb R, Borsari AC, Rosa-e-Silva ACJ, et al. Brief cognitive behavioral intervention in groups in a Brazilian assisted reproduction program. J Behav Med 2012;38(2):29-35. DOI: 10.1080/0896428.2012. 65483.

2. Deka PK, Sarma S. Psychological aspects of infertility. Br J Med Pract 2010;3(3):336.

3. Vander Borght M, Wyns C. Fertility and infertility: definition and epidemiology. Clin Biochem 2018;62:2-10. DOI: 10.1016/j. clinbiochem.2018.03.012.

4. Tao P, Coates R, Maycock B. The impact of infertility on sexuality: a literature review. Med J Aust 2011;4(11):620. DOI: 10.4066/ AMJ.20111055.

5. Jamilian M, Rafiei M, Jamilian HR, et al. The comparison of general health between fertile and infertile women of Arak City in 2010. J Arak Univer Med Sci 2012;14(6):27-35.

6. Keyes $C L$, Shmotkin D, Ryff CD. Optimizing well-being: the empirical encounter of two traditions. J Pers Soc Psychol 2002;82(6):1007. DOI: $10.2307 / 3090197$.

7. Küchenhoff J. Unfulfilled desire for children--what is the grief for men? Therapeutische Umschau. Revue Therapeuti 1999;56(5):260-264. DOI: $10.1024 / 40-5930.56 .5 .260$.

8. Ryan RM, Huta V, Deci EL. Living well: a self-determination theory perspective on eudaimonia. J Happiness Stud 2008;9(1):139-170. DOI: 10.1007/S10902-006-9023-4.

9. Zaeri Amirany M, Pourshahriari MS, Beh-pajooh A. The impact of humor training on marital adjustment and psychological well-being in female teachers in ardestsn city. Psychol Stud 2016;12(1):85-106. DOI: $10.22051 /$ psy.2016.2257.

10. Safarinia M, Aghayousefi A, Baradaran M. The relationship between personality aspects, problem solving and psychological well-being the role of narcissism. Counsel Psychot Cult 2014;5:89-102.

11. Eid M, Larsen RJ. The science of subjective well-being. Guilford Press; 2008.

12. Rosen JC, Orosan P, Reiter J. Cognitive behavior therapy for negative body image in obese women. Behav Ther 1995;26(1):25-42. DOI: 10/1016/s0005-7894(05)80081-4.

13. Berg RC, Landreth GL, Fall KA. Group counseling: concepts and procedures. 4th ed., Routledge; 2013.

14. Ahmadi Z, Abbaspoor Z, Behroozy N, et al. The effects of cognitive behavioral therapy on body image in infertile women. Iran Red Crescent 2017;19(10). DOI: doi.org/10.5812/ircmj.14903.

15. Mosalanejad L, Koolaee AK, Jamali S. Effect of cognitive behavioral therapy in mental health and hardiness of infertile women receiving assisted reproductive therapy (ART). Iran J Reprod Med 2012;10(5): 483.

16. Talaei A, Kimiaei SA, Borhani Moghani M, et al. Effectiveness of group cognitive behavioral therapy on depression in infertile women. Iranian J Obstet, Gynecol Infertil 2014;17(94):1-9.

17. Bavadi B, Poursharifi H, Lotfikashani F. The effectiveness of cognitivebehavioral therapy based 8-step pattern of cash to improve psychological well-being of women with breast cancer. Iran J Breast Dis 2016;8(4):41-51.

18. Nekouei ZK, Yousefy A, Manshaee GH. Cognitive-behavioral therapy and quality of life: an experience among cardiac patients. J Educ Health Promot 2012;1(2):48-62. DOI: 10.4103/2277-95310. 94410.

19. Norbalah AA, Ramadanzadeh F, Abedinia N, et al. Prevalence of psychiatric disorders and type personality in fertile and infertile women. J Reprod Infertil 2009;9(4):350-360.

20. Khanjani M, Shahidi SH, Fath-Abadi J, et al. Factor structure and psychometric properties of the Ryff's scale of psychological wellbeing, short form (18-item) among male and female students. JTBCP 2014;8(32):27-36.

21. Hayes SC, Hofmann SG. Process-based CBT: The science and core clinical competencies of cognitive behavioral therapy. New Harbinger Publications; 2018. 
22. Hashemi N, Ghayour Baghbani SM, Khadivi G, et al. The effectiveness of cognitive behavioral therapy and schema therapy on happiness and mental health of nursing students. J Clin Nurs Midwi 2016;5(1): $12-22$.

23. Roothman B, Kirsten DK, Wissing MP. Gender differences in aspects of psychological well-being. S Afr J Psychol 2003;33(4):212-218. DOI: 10.1177/008124630303300403.

24. Faramarzi M, Pasha H, Esmailzadeh S, et al. The effect of the cognitive behavioral therapy and pharmacotherapy on infertility stress: a randomized controlled trial. Int J Fertil Steril 2013;7(3):199-206.
25. Abdolahi HM, Ghojazadeh M, Abdi S, et al. Effect of cognitive behavioral therapy on anxiety and depression of infertile women: a meta-analysis. Asian Pac J Reprod 2019;8(6):251-259. DOI: 10.4103/2305-0500.270102.

26. Hamzeh Pour T. The effect of cognitive behavioral therapy on anxiety in infertile women. Euro J Exp Biol 2014;4(1):415-419.

27. Espie CA, Emsley R, Kyle SD, et al. Effect of digital cognitive behavioral therapy for insomnia on health, psychological well-being, and sleeprelated quality of life: a randomized clinical trial. JAMA Psychiatry 2019;76(1):21-30. DOI: 10.1001/jamapsychiatry.2018.2745. 